\title{
Categories Related to Topology Viewed as Soft Sets
}

\author{
Banu Pazar Varol ${ }^{1}$ Alexander Shostak ${ }^{2}$ Halis Aygün ${ }^{3}$ \\ ${ }^{1,3}$ Department of Mathematics, University of Kocaeli, Umuttepe Campus, 41380, Kocaeli - TURKEY \\ ${ }^{2}$ Department of Mathematics, University of Latvia, LV-1002, Riga - LATVIA
}

\begin{abstract}
D. Molodtsov (1999) introduced the concept of a soft set as a new approach for modeling uncertainties. The aim of this work is to define special kinds of soft sets, namely soft, $L$-fuzzifying soft, $L$-soft, and $L$-fuzzy soft neighborhood sets and to use them in order to give an alternative characterization of categories related to topology: crisp topological, $L$-topological, $L$-fuzzifying topological and $L$-fuzzy topological spaces.
\end{abstract}

Keywords: soft set, soft neighborhood set, $L$ fuzzifying soft neighborhood set, $L$-soft neighborhood set, $L$-fuzzy soft neighborhood set, topology.

\section{Introduction}

In 1999 D. Molodtsov [12] introduced the concept of a soft set and started to develop basics of the the corresponding theory as a new approach for modeling uncertainties. The aim of this notion was to make a certain discretization of such fundamental mathematical concepts with essentially continuous nature as a limit, continuity, a derivative, an integral, etc., thus providing new tools for the use of the machinery of mathematical analysis in applications. To achieve this aim a certain parametrization of a given set $X$ was proposed thus resulting in the concept of a soft structure over the set $X$.

As usually, new perspective ideas draw attention both of mathematicians working in the field of pure mathematics as well as of researchers in the areas of applied mathematics. Specifically, the specialists found the concept of a soft set well coordinated with such modern mathematical concepts as a fuzzy set and a more general, multi-valued set. These ideas have resulted in a series of works where soft versions of fuzzy mathematical concepts were realized. In particular, the concepts of soft topologies [3], fuzzy soft topologies [14], soft rings [1], fuzzy soft rings [15] etc., were introduced. On the other hand the aim of this work is not a generalization of classical mathematical concepts in the frames of the theory of soft sets, but rather a reinterpretation of known, non-soft mathematical concepts by means of terminology borrowed from the theory of soft sets. To be more precise, in this work we are interested to interpret categories related to topological spaces ( namely, crisp topological spaces, $L$ - topological spaces ([4], [6]), $L$-fuzzifying topological spaces ([7], [21]) and $L$-fuzzy topological spaces ([9], [19], [20])) as special categories of soft sets. The restricted volume of this work does not allow us to give proofs - there is only one theorem (Theorem 3.12) given with proof in order to illustrate how the machinery of soft sets can be used in the study of these categories. The detailed research of these categories and some other categories defined on the basis of soft neighborhood sets will be considered in a paper which is now in preparation.

\section{Preliminaries}

Throughout this paper, let $X$ be a nonempty set refereed to as the universe, and let $E$ be the set of all parameters for the universe $X$.

Definition 2.1 (cf $[12,13])$. Let $X$ be a universe set and $E$ be a set of parameters. A pair $(M, E)$ is called a soft set over $X$ if $M$ is a mapping from $E$ into the set of all subsets of the set $X$, i.e., $M: E \longrightarrow 2^{X}$. In what follows we denote a soft set $(M, E)$ over $X$ as a triple $(M, E, X)$. The mapping $M: E \rightarrow 2^{X}$ will be referred to as a soft structure on the set $X$ and the triple $(M, E, X)$ will be referred just as a soft set.

\section{Remark 2.2 [Soft sets versus fuzzy sets]}

The concept of a soft set is sometimes compared to the concept of a fuzzy set, or more generally, to the concept of an $L$-fuzzy set. Indeed, these concepts have some unifying features. Both of them were "invented" to make "a revision" of the classical fundamental mathematical concept of a set in order to make it more suitable for describing applied problems, in particular, to deal with ill-posed problems, and to develop mathematical models for processes inherently having uncertainty in their description. Moreover, there is also obvious similarity in the formal definitions of soft sets and $L$-fuzzy sets. Indeed, let $A: X \rightarrow L$ be an $L$-fuzzy subset of a set $X$. Defining for each $\alpha \in L$ a subset $A_{\alpha}=\{x \mid A(x) \geq \alpha\} \subseteq X$ we obtain a level decomposition (sometimes called the horizontal representation [5]) of the fuzzy set $A$ :

$$
A: X \rightarrow L \Longleftrightarrow\left\{A_{\alpha} \mid \alpha \in L\right\} .
$$

Thus actually an $L$-fuzzy set $A$ is realized as a soft structure $M_{A}: L \rightarrow 2^{X}$ where $M_{A}(\alpha)=A_{\alpha}$ and 
the set of parameters is realized by the lattice $L$, cf also [16]. On the other hand having a soft structure $M: E \rightarrow 2^{X}$ on $X$, sometimes it is possible to interpret it as a horizontal representation of an $E$-fuzzy subset $A_{M}: E \rightarrow 2^{X}$ of the set $X$. (We can not go into the details here). However the analogy between the concepts of fuzzy sets and soft sets is quite limited. Concerning the philosophical backgrounds of the two concepts, the essential difference is that fuzzy sets are based on the concept of the degree of belongness, thus making use of a certain "continuous version" of the belongness predicate. On the other hand the idea of a soft set, as it was already mentioned in the Introduction, is the opposite one - namely, to make a certain discretization of inherently continuous concepts of analysis. Further, concerning the formal part of the definitions. The definition of a fuzzy set essentially relies on the structure of complete lattices - the subsets of $X$ in the horizontal representation of an $L$-fuzzy set $A$ are ordered by partial order realized by inclusion and besides the bottom $X=A_{0}$ and the top $A_{1}$ elements are inherently present in this representation. On the other hand the set of parameters used in the definition of a soft set need not have (and usually indeed does not have) any structure and therefore parametrized by the elements $e \in E$ subsets $M(e)$ of $X$ need not be related.

Definition 2.3 Given two soft structures $M_{1}$ : $E \rightarrow 2^{X}, M_{2}: E \rightarrow 2^{X}$ over the set $X$ we say that $M_{1}$ is weaker than $M_{2}$ (and $M_{2}$ is stronger than $M_{1}$ ) if $M_{1}(e) \subseteq M_{2}(e)$ for every $e \in E$. We write in this case $M_{1} \preceq M_{2}$.

In what follows we shall often use the following statement the proof of which is straightforward:

\section{Theorem 2.4 [Lattice of soft structures]}

Let a pair $(E, X)$ be given and let $\mathcal{M}$ be the set of all soft structures $M: E \rightarrow 2^{X}$ on the set $X$ equipped with the partial order $\preceq$. Then $(\mathcal{M}, \preceq)$ is a complete lattice where the supremum and the infimum of a family $\left\{M_{i} \mid i \in I\right\}$ are defined respectively by $\bigvee_{i \in I} M_{i}(e)=\bigcup_{i \in I} M_{i}(e)$ and $\bigwedge_{i \in I} M_{i}(e)=$ $\bigcap_{i \in I} M_{i}(e)$. In particular the top and the bottom elements in the lattice $\mathcal{M}$ are given respectively by $\mathcal{M}_{\top}(e)=X \forall e \in E$ and $\mathcal{M}_{\perp}(e)=\emptyset \forall e \in E$.

To consider soft sets as a category we have to specify morphisms between two soft sets.

Definition 2.5 Let $\psi: E \rightarrow F$ and $\varphi: X \rightarrow Y$ be two functions. Then the pair $(\psi, \varphi):(M, E, X) \rightarrow$ $(N, F, Y)$ is called a soft mapping from $(M, E, X)$ to $(N, F, Y)$ if $\varphi \rightarrow \circ \succeq N \circ \psi$ :

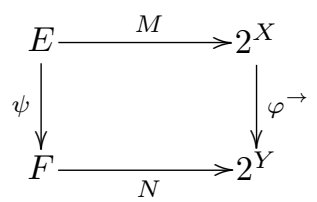

where $\varphi^{\rightarrow}: 2^{X} \rightarrow 2^{Y}$ is the forward powerset operator induced by the mapping $\varphi$ (see e.g. [18]), that is $\varphi^{\rightarrow}(A):=\varphi(A)$ for every $A \in 2^{X}$.

Remark 2.6 Note that in [3] a soft function from $(M, E, X)$ to $(N, F, Y)$ is defined just as a pair of functions $(\psi, \varphi):(M, E, X) \rightarrow(N, F, Y)$ without specifying any relations between mappings $\varphi: X \rightarrow$ $Y$ and $\psi: E \rightarrow F$. In our opinion this does not allow to include soft sets in the reasonable categorical frames, therefore we redefine this definition as above.

Since componentwise composition of two soft mappings $(\psi, \varphi):(M, E, X) \rightarrow(N, F, Y)$ and $\left(\psi^{\prime}, \varphi^{\prime}\right):(N, F, Y) \rightarrow(P, G, Z)$ is obviously a soft mapping

$$
\left(\psi^{\prime} \circ \psi, \varphi^{\prime} \circ \varphi\right):(M, E, X) \rightarrow(P, G, Z)
$$

and the pair of identities $\left(i d_{E}, i d_{X}\right):(M, E, X) \rightarrow$ $(M, E, X)$ is the identical soft mapping, soft sets and soft mappings between them form a category which will be denoted SOFSET.

Let $(M, E, X)$ and $(N, F, Y)$ be two soft sets and $(\psi, \varphi)$ be a soft function from $(M, E, X)$ to $(N, F, Y)$. The image of $(M, E, X)$ under the soft function $(\psi, \varphi)$ is defined by $(\psi, \varphi)(M, E, X):=$ $(\varphi(M), \psi(E), Y)$, where $\psi(E)$ is the image of $E$ in the category SET and $\varphi(M)$ is defined by the Zadeh extension principle, that is

$$
\varphi^{\rightarrow}(M)(f)=\bigcup_{\psi(e)=f} \varphi(M(e)), \quad \forall f \in F
$$

see e.g. [3].

The pre-image of $(N, F, Y)$ under the soft function $(\psi, \varphi)$ is defined by

$$
(\psi, \varphi)^{-1}(N, F, Y):=\left(\varphi^{\leftarrow} \circ N \circ \psi, \psi^{-1}(F), X\right)
$$

where $\psi^{-1}(F)$ is the preimage of $F$ in the category of sets and the mapping $\varphi^{\leftarrow}$ is the backward operator induced by the mapping $\varphi: X \rightarrow Y$, (see e.g. [18]) that is

$$
\left(\varphi^{\leftarrow} \circ N \circ \psi\right)(e)=\varphi^{\leftarrow}(N(\psi(e))), \quad \forall e \in \psi^{-1}(F),
$$

cf e.g. [3]

Definition 2.7 For two soft sets $(M, E, X)$ and $(N, F, Y)$, we say that $(M, E, X)$ is a soft subset of $(N, F, Y)$ and write $(M, E, X) \sqsubseteq(N, F, Y)$ if

(i) $X \subseteq Y$

(ii) $E \subseteq F$ and

(iii) For each $e \in E, M(e)=N(e) \cap X$.

Two soft sets $(M, E, X)$ and $(N, F, Y)$ are said to be equal if $(M, E, X) \sqsubseteq(N, F, Y)$ and $(N, F, Y) \sqsubseteq$ $(M, E, X)$. 
Remark 2.8 From Definition 2.7 and Theorem 2.4 it is clear that the soft subset $(M, E, X)$ of a soft set $(N, F, Y)$ can be characterized as the initial soft structure $M: E \rightarrow 2^{X}$ for the mapping $(\psi, \varphi)$ : $(E, X) \rightarrow(N, F, Y)$ determined by a pair of inclusion functions $\varphi: X \rightarrow Y$ and $\psi: E \rightarrow F$ in the category SOFSET. Hence a soft subset $(M, E, X)$ of a soft set $(N, F, Y)$ is indeed its subobject in the category SOFSET.

Definition 2.9 [Construction of the product] Given two soft sets $(M, E, X)$ and $(N, F, Y)$ we consider the triple $(M \times N, E \times F, X \times Y)$ where the mapping $M \times N: E \times F \rightarrow 2^{X \times Y}$ is defined by

$(M \times N)(e, f)=M(e) \times N(f) \in 2^{X} \times 2^{Y} \subseteq 2^{X \times Y}$.

One can easily see that the pairs of projections $p_{E}$ : $E \times F \rightarrow E, q_{X}: X \times Y \rightarrow X$ and $p_{F}: E \times F \rightarrow F$, $q_{Y}: X \times Y \rightarrow Y$ determine morphisms

$\left(p_{E}, q_{X}\right):(M \times N, E \times F, X \times Y) \rightarrow(M, E, X)$ and

$\left(p_{F}, q_{Y}\right):(M \times N, E \times F, X \times Y) \rightarrow(N, F, Y)$.

Further, let $\mathcal{P}$ be the family of all soft structures $P$ on $(E \times F, X \times Y)$ for which

$$
\begin{gathered}
\left(p_{E}, q_{X}\right):(P, E \times F, X \times Y) \rightarrow(M, E, X) \text { and } \\
\left(p_{F}, q_{Y}\right):(P, E \times F, X \times Y) \rightarrow(N, F, Y)
\end{gathered}
$$

are morphisms in the category SOFSET and let $P_{0}:=\bigwedge \mathcal{P}$. Then $\left(P_{0}, E \times F, X \times Y\right)$ is the product of soft sets $(M, E, X)$ and $(N, F, Y)$ in the category

\section{SOFSET}

Definition 2.10 [Construction of the coproduct] Given two soft sets $(M, E, X)$ and $(N, F, Y)$ we define their coproduct as the triple $(M \oplus N, E \oplus$ $F, X \oplus Y$ ) where $E \oplus F$ and $X \oplus Y$ are discrete sums of the corresponding sets and the mapping

$$
M \oplus N: E \oplus F \rightarrow 2^{X} \oplus 2^{Y} \subseteq 2^{X \oplus Y}
$$

is defined by $(M \oplus N)(e)=M(e) \in 2^{X \oplus Y}$ if $e \in E$ and $(M \oplus N)(f)=N(f) \in 2^{X \oplus Y}$.

One can easily see that the pairs of inclusions $i_{E}$ : $E \rightarrow E \oplus F, j_{X}: X \rightarrow X \oplus Y$ and $i_{F}: F \rightarrow E \oplus F$, $j_{Y}: Y \rightarrow X \oplus Y$ determine morphisms

$$
\begin{gathered}
\left(i_{E}, j_{X}\right):(M, E, X) \rightarrow(M \oplus N, E \oplus F, X \oplus Y) \text { and } \\
\left(i_{F}, j_{Y}\right):(N, F, Y) \rightarrow(M \oplus N, E \oplus F, X \oplus Y)
\end{gathered}
$$

and that coproduct defined above is indeed the coproduct in the category of soft sets.

\section{Soft neighborhood sets}

In this section we characterize the category of topological spaces TOP as a certain subcategory of the category SOFSET and discuss relations between the two categories. Throughout this section, given a set $X$ we take it as the set of parameters and the exponent $2^{X}$ as the set over which the soft structure is being constructed. Thus all soft sets considered here are described by triples $\left(M, X, 2^{X}\right)$.
Definition 3.1 Let $\left(\mathcal{U}, X, 2^{X}\right)$ be a soft set, i.e. $\mathcal{U}: X \rightarrow 2^{2^{X}}$. We call $\mathcal{U}: X \rightarrow 2^{2^{X}}$ a soft neighborhood structure on $X$ and the triple $\left(\mathcal{U}, X, 2^{X}\right)$ a soft neighborhood set if for every $x \in X$ the family $\mathcal{U}(x):=\mathcal{U}_{x} \in 2^{2^{X}}$ satisfies the following conditions:

(N1) If $U \in \mathcal{U}(x)$, then $x \in U$;

(N2) If $U \in \mathcal{U}(x)$ and $U \subset V$, then $V \in \mathcal{U}(x)$;

(N3) If $U, V \in \mathcal{U}(x)$, then $U \cap V \in \mathcal{U}(x)$;

(N4) If $U \in \mathcal{U}(x)$, then there exists $V \in \mathcal{U}(x)$ such that $V \in \mathcal{U}(y)$ whenever $y \in V$.

Let NSOFSET denote the category of soft neighborhood sets, that is the full subcategory of the category SOFSET of soft sets and soft mapping, whose objects are soft neighborhood sets.

Theorem 3.2 Let $(X, \mathcal{T})$ be a topological space and for each $x \in X$ let $\mathfrak{U}_{x}$ be the family of neighborhoods of a point $x$ in this space. Further, define the mapping $\mathcal{U}_{\mathcal{T}}: X \rightarrow 2^{2^{X}}$ by $\mathcal{U}_{\mathcal{T}}(x)=$ $\mathfrak{U}_{x}$. Then $\left(\mathcal{U}_{\mathcal{T}}, X, 2^{X}\right)$ is a soft neighborhood set. We call this soft neighborhood structure $\mathcal{U}_{\mathcal{T}}$ generated by the topology $\mathcal{T}$ and the soft neighborhood set $\left(\mathcal{U}_{\mathcal{T}}, X, 2^{2^{X}}\right)$ generated by the topological space $(X, \mathcal{T})$.

Conversely, given a soft neighborhood set $\left(\mathcal{U}, X, 2^{X}\right)$ we define a family $\mathcal{T}_{\mathcal{U}}$ of subsets of $X$ by taking all $U \subseteq X$ such that $U \in \mathcal{U}(x)$ whenever $x \in U$. Then $\mathcal{T}_{\mathcal{u}}$ is a topology on the set $X$ and $\{\mathcal{U}(x) \mid x \in X\}$ is exactly the family of all neighborhoods of a point $x$ in this space. We say that the topology $\mathcal{T}_{\mathcal{U}}$ is generated by the soft structure $\mathcal{U}$. and the topological space $\left(X, \mathcal{T}_{\mathfrak{U}}\right)$ generated by the soft neighborhood set $\left(\mathcal{U}, X, 2^{X}\right)$.

Besides $\mathcal{U}_{\mathcal{T}_{u}}=\mathcal{U}$ for any soft neighborhood structure $\mathcal{U}$ on a set $X$ and $\mathcal{T}_{\mathcal{U}_{\mathcal{T}}}=\mathcal{T}$ for any topology $\mathcal{T}$ on a set $X$.

Note that the proof of this (important in the context of our work) theorem can be obtained just as a reformulation in terms of soft sets of the well-known characterization of topologies by means of neighborhoods (see e.g. [8]).

In what follows we need to use the order $\preceq$ introduced in Definition 2.3 in case when we deal with soft neighborhood structures $\mathcal{U}: X \rightarrow 2^{2^{X}}$. Therefore we analyze the relation $\preceq$ in this special case. Note first that taking into account axiom (N2) in Definition 3.1 for a fixed $x \in X$ we have

$\mathcal{U}_{x} \supseteq \mathcal{V}_{x} \Leftrightarrow \forall V_{x} \in \mathcal{V}_{x} \exists U_{x} \in \mathcal{U}_{x}$ such that $U_{x} \subseteq V_{x}$.

Hence the relation $\preceq$ on the set of soft neighborhood structures

$$
\mathfrak{U}(X):=\left\{\mathcal{U} \mid \mathcal{U}: X \rightarrow 2^{2^{X}}\right\}
$$

can be realized as follows: 


$$
\begin{gathered}
\mathcal{V} \preceq \mathcal{U} \Leftrightarrow\left(\forall x \in X, \forall V_{x} \in \mathcal{V}_{x}\right. \\
\left.\exists U_{x} \in \mathcal{U}_{x} \text { such that } U_{x} \subseteq V_{x}\right) .
\end{gathered}
$$

In its turn, referring to Theorem 3.2, this allows to characterize continuity of a mapping $\psi$ : $\left(X, \mathcal{T}_{X}\right) \rightarrow\left(Y, \mathcal{T}_{Y}\right)$ by the property

$\left(\psi^{\rightarrow}\right) \rightarrow \circ \mathfrak{U} \succeq \mathcal{V} \circ \psi$, where $\mathcal{U}:=\mathcal{U}_{\mathcal{T}_{X}}, \mathcal{V}:=\mathcal{V}_{T_{Y}}$

see the diagrams:
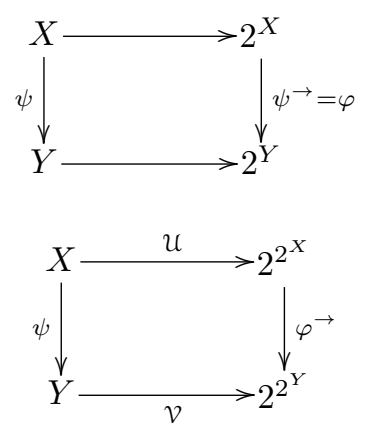

We summarize the obtained information as follows:

Theorem 3.3 Given topological spaces $\left(X, \mathcal{T}_{X}\right)$ and $\left(Y, \mathcal{T}_{Y}\right)$, let $\psi: X \rightarrow Y$ be a mapping of the corresponding sets. Then the mapping $\psi:\left(X, \mathcal{T}_{X}\right) \rightarrow$ $\left(Y, \mathcal{T}_{Y}\right)$ is continuous, i.e. a morphism in the category TOP, if and only if

$$
\left(\psi,\left(\psi^{\rightarrow}\right) \rightarrow\right):\left(\mathcal{U}_{\mathcal{T}_{X}}, X, 2^{X}\right) \rightarrow\left(\mathcal{U}_{\mathcal{T}_{Y}}, Y, 2^{Y}\right)
$$

is a soft mapping of the corresponding soft neighborhood sets, that is a morphism in the category

\section{NSOFSET.}

From Theorems 3.2 and 3.3 we get

Corollary 3.4 Functor $\Phi:$ TOP $\rightarrow$ NSOFSET which assigns to a topological space $(X, \mathcal{T})$ the generated soft neighborhood set $\left(\mathcal{U}_{\mathcal{T}}, X, 2^{X}\right)$ and assigns to a continuous mapping $\psi:\left(X, \mathcal{T}_{X}\right) \rightarrow\left(Y, \mathcal{T}_{Y}\right)$ the soft mapping

$$
\left(\psi,\left(\psi^{\rightarrow}\right) \rightarrow\right):\left(\mathcal{U}_{\mathcal{T}_{X}}, X, 2^{X}\right) \rightarrow\left(\mathcal{U}_{\mathcal{T}_{Y}}, Y, 2^{Y}\right)
$$

is an isomorphism.

Let $\Psi:$ NSOFSET $\rightarrow$ TOP denote the inverse of functor $\Phi$.

Taking into account constructions of a subset and the product of soft sets 2.9, 2.7 and the description of a topological space as a soft neighborhood set given in the previous corollary 3.4 , we obtain the following three propositions:

Proposition 3.5 Let $\left(\mathcal{U}, X, 2^{X}\right)$ be a soft neighborhood set and $A \subseteq X$. Consider the mapping $\mathcal{U}_{A}: A \rightarrow 2^{2^{A}}$ defined by $\mathcal{U}_{A}(a)=A \cap \mathcal{U}(a)$ for each $a \in A$. The family $\mathcal{U}_{A}(x)=\{A \cap \mathcal{U}(x) \mid x \in A\}$ satisfies conditions (N1)-(N4) and $\left(\mathcal{U}_{A}, A, 2^{A}\right)$ is a soft neighborhood subset of the soft neighborhood set $\left(\mathcal{U}, X, 2^{X}\right)$.
Proposition 3.6 Let $\left(\mathcal{U}, X, 2^{X}\right)$ and $\left(\mathcal{V}, Y, 2^{Y}\right)$ be soft neighborhood sets. Then their product $(\mathcal{W}, X \times$ $Y, 2^{X \times Y}$ ) in the category NSOFSET is a soft neighborhood set and the corresponding topological space $\left(X \times Y, \mathcal{T}_{\mathcal{W}}\right)$ is the product of the topological spaces $\left(X, \mathcal{T}_{\mathcal{U}}\right)$ and $\left(Y, \mathcal{T}_{\mathcal{V}}\right)$ in the category $\mathbf{T O P}$.

Proposition 3.7 Let $\left(\mathcal{U}, X, 2^{X}\right)$ and $\left(\mathcal{V}, Y, 2^{Y}\right)$ be soft neighborhood sets. Then the coproduct $(\mathcal{W}, X \oplus$ $Y, 2^{X \oplus Y}$ ) in the category NSOFSET is a soft neighborhood set and the corresponding topological space $\left(X \oplus Y, \mathcal{T}_{\mathcal{W}}\right)$ is the direct sum of the topological spaces $\left(X, \mathcal{T}_{\mathcal{U}}\right)$ and $\left(Y, \mathcal{T}_{\mathcal{V}}\right)$ in the category TOP.

Example 3.8 Let $\left(U, \mathbb{R}, 2^{\mathbb{R}}\right)$ be the ordinary real line $\mathbb{R}$ viewed as a soft neighborhood set and let $A \subset$ $\mathbb{R}$. Then the soft neighborhood subset $\left(\mathcal{U}_{A}, A, 2^{A}\right)$ of $\left(\mathcal{U}, \mathbb{R}, 2^{\mathbb{R}}\right)$ corresponds to the subspace $A$ of $\mathbb{R}$. The product of soft neighborhood sets

$$
\left(\mathcal{U}, \mathbb{R}, 2^{\mathbb{R}}\right) \times\left(\mathcal{U}, \mathbb{R}, 2^{\mathbb{R}}\right)
$$

is a soft neighborhood set whose topological counterpart is the plane $\mathbb{R}^{2}$.

Definitions of image and pre-image of a soft set in case of a soft neighborhood set can be redefined as follows:

Let $(\psi,(\psi \rightarrow) \rightarrow):\left(\mathcal{U}, X, 2^{X}\right) \rightarrow\left(\mathcal{V}, Y, 2^{Y}\right)$ be a soft mapping, that is a morphism in the category NSOFSET

(1) The image of $\left(\mathcal{U}, X, 2^{X}\right)$ under the soft mapping $(\psi,(\psi \rightarrow) \rightarrow)$ is the soft neighborhood set on $2^{Y}$ defined by

$\left(\psi,\left(\psi^{\rightarrow}\right)^{\rightarrow}\right)\left(\mathcal{U}, X, 2^{X}\right)=\left(\left(\left(\psi^{\rightarrow}\right) \rightarrow\right)(\mathcal{U}), \psi(X), 2^{Y}\right)$,

where

$$
\left(\left(\psi^{\rightarrow}\right) \rightarrow\right)(\mathcal{U})(y)=\bigcup_{\psi(x)=y} \psi(\mathcal{U}(x)), \quad \forall y \in Y .
$$

(2) The pre-image of $\left(\mathcal{V}, Y, 2^{Y}\right)$ under the soft mapping $(\psi,(\psi \rightarrow) \rightarrow)$ is the soft neighborhood set

$\left(\psi,\left(\psi^{\rightarrow}\right)^{\rightarrow}\right)^{-1}\left(\mathcal{V}, Y, 2^{Y}\right)=\left(\left(\psi^{\rightarrow}\right)^{\leftarrow} \circ \mathcal{V} \circ \psi, \psi^{-1}(Y), 2^{X}\right)$, see the diagram:

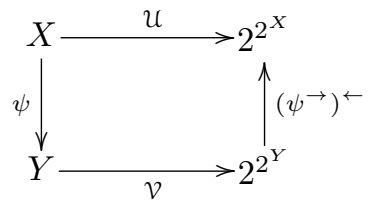

\subsection{Topological properties of soft neighborhood sets}

Since there is a natural isomorphism $\Psi$ between the category NSOFSET and the category TOP of topological spaces, one can introduce the following general definition: 
Definition 3.9 Let $\mathcal{P}$ be a topological property. We say that a soft neighborhood set $\left(\mathcal{U}, X, 2^{X}\right)$ has property $\mathcal{P}$ if and only if the topological space $\left(X, \mathcal{T}_{\mathcal{U}}\right)=\Psi\left(\mathcal{U}, X, 2^{X}\right)$ has property $\mathcal{P}$.

However in some cases it is possible to obtain "nice" characterizations of topological properties of soft neighborhood sets in the terminology of NSOFSET, without applying their counterparts in the category TOP. As the first example we characterize lower separation axioms in soft neighborhood sets.

Theorem 3.10 Let $\left(\mathcal{U}, X, 2^{X}\right)$ be a soft neighborhood set. Then

1. $\left(\mathcal{U}, X, 2^{X}\right)$ is a $T_{0}$-soft neighborhood set if and only if for all $x, y \in X, x \neq y$, it holds

$$
\mathcal{U}(x) \neq \mathcal{U}(y) .
$$

2. (U, $\left.X, 2^{X}\right)$ is a $T_{1}$-soft neighborhood set if and only if for all $x, y \in X, x \neq y$, it holds

$$
\mathcal{U}(x) \backslash \mathcal{U}(y) \neq \emptyset .
$$

3. $\left(\mathcal{U}, X, 2^{X}\right)$ is a $T_{2}$-soft neighborhood set if and only if for all $x, y \in X, x \neq y$, there exist $U_{x} \in$ $\mathcal{U}(x)$ and $U_{y} \in \mathcal{U}(y)$ such that $U_{x} \cap U_{y}=\emptyset$.

To characterize compactness in the terms of soft neighborhood sets it is convenient to introduce the notion of a choice function in a soft neighborhood set $\left(\mathcal{U}, X, 2^{X}\right)$. Namely by it we mean a function $c: X \rightarrow 2^{X}$ such that $c(x) \in \mathcal{U}(x)$ for each $x \in X$. Given a set $A \subseteq X$ we denote $c(A)=\bigcup_{x \in A} c(x)$. Obviously $c(X)=X$ for each choice function. Now it is clear that compactness can be characterized as follows:

Theorem 3.11 A soft neighborhood set $\left(\mathcal{U}, X, 2^{X}\right)$ is compact if only if for each choice function $c: X \rightarrow 2^{X}$ there exists a finite set $X_{c}=$ $\left\{x_{1}, \ldots, x_{n}\right\} \subseteq X$ such that $c\left(X_{c}\right)=X$.

To illustrate the work of this characterization of compactness in the context of soft neighborhood sets we give a "soft proof" of the well-known statement that a continuous image of a compact space is compact.

Theorem 3.12 Let $\left(\mathcal{U}, X, 2^{X}\right)$ and $\left(\mathcal{V}, Y, 2^{Y}\right)$ be two soft neighborhood sets and $\left(\mathcal{U}, X, 2^{X}\right)$ be compact. If $\psi: X \rightarrow Y$ is surjective and $(\psi,(\psi \rightarrow) \rightarrow)$ : $\left(\mathcal{U}, X, 2^{X}\right) \rightarrow\left(\mathcal{V}, Y, 2^{Y}\right)$ is a soft mapping, then $\left(\mathcal{V}, Y, 2^{Y}\right)$ is compact.

Proof. Let $c_{Y}: Y \rightarrow 2^{Y}$ be a choice function in the soft neighborhood set $\left(\mathcal{V}, Y, 2^{Y}\right)$. Then by defining $c_{X}: X \rightarrow 2^{X}$ as $c_{X}(x):=\psi^{\leftarrow} \circ c_{Y} \circ \psi(x)$ we get a commutative diagram:

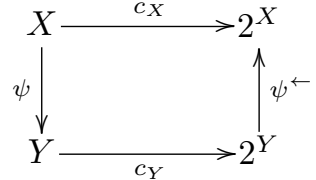

Since $\left(\psi,\left(\psi^{\rightarrow}\right) \rightarrow\right):\left(\mathcal{U}, X, 2^{X}\right) \rightarrow\left(\mathcal{V}, Y, 2^{Y}\right)$ is a soft mapping and since $c_{Y}(y) \in \mathcal{V}(y)$ for each $y \in Y$, we conclude that

$c_{X}(x)=\psi^{\leftarrow} \circ c_{Y} \circ \psi(x)=\psi^{\leftarrow}\left(c_{Y}(y)\right) \in \mathcal{U}(x)$,

and hence $c_{X}$ is a choice function in $\left(\mathcal{U}, X, 2^{X}\right)$.

By the compactness of $\left(\mathcal{U}, X, 2^{X}\right)$ there exists a finite subset $X_{c} \subseteq X$ such that $c_{X}\left(X_{c}\right)=X$. We define $Y_{c}=\psi\left(X_{c}\right)$. Then taking into account that $\psi$ is an onto mapping we get

$c_{Y}\left(Y_{c}\right)=c_{Y}\left(\psi\left(X_{c}\right)\right)=\left(\psi^{\rightarrow} \circ c_{X} \circ \psi^{\leftarrow}\right)\left(\psi\left(X_{c}\right)\right)=Y$

and hence $\left(\mathcal{V}, Y, 2^{Y}\right)$ is compact.

\section{L-Fuzzifying soft neigborhood set}

\section{1. $L$-fuzzy soft sets over a set $X$}

Throughout Section 4, Section 5 and Section $6, L=$ $\left(L, \leq, \vee, \wedge,{ }^{\prime}\right)$ denotes a fuzzy lattice, i.e., a completely distributive complete lattice with an orderreversing involution '. Further, $1_{L}$ and $0_{L}$ denote the top and the bottom elements of $L$, respectively. Let $L^{X}$ be the set of all $L$-fuzzy sets on $X$ and let $M(L)$ denote the set of all non-zero coprimes in $L$, that is $\lambda \in M(L)$ if and only if $\lambda^{\prime}$ is prime and $\lambda^{\prime} \neq 1_{L}$.

Definition 4.1 A triple $(m, E, X)$ is called an $L$ fuzzy soft set and the mapping $m: E \rightarrow L^{X}$ an $L$-fuzzy soft structure on the set $X$. Thus for each $e \in E, m(e)=m_{e}: X \rightarrow L$ is an $L$-fuzzy subset of $X$.

If we take $L=I=[0,1]$ and $A \subset E$, then $(m, A, X)$ is a fuzzy soft set on $X$ as it is defined by Maji et. al. [11].

Definition 4.2 Given two L-fuzzy soft structures $m_{1}, m_{2}: E \rightarrow L^{X}$ on a set $X$ we say that $m_{1}$ is weaker than $m_{2}$ (respectively, $m_{2}$ is stronger than $\left.m_{1}\right)$ if $m_{1}(e) \leqslant m_{2}(e)$ for every $e \in E$. We write in this case $m_{1} \preceq m_{2}$.

\section{Theorem 4.3 [Lattice of fuzzy soft struc- tures]}

Let a pair $(E, X)$ be given and let $\mathcal{M}$ be the set of all $L$-fuzzy soft structures $m: E \rightarrow L^{X}$ on the set $X$ equipped with the partial order $\preceq$. Then $(\mathcal{M}, \preceq)$ is a complete lattice where the supremum, the infimum and order-reversing involution of a family $\left\{m_{i} \mid i \in I\right\}$ are defined respectively by $\bigvee_{i \in I} m_{i}(e)$, $\bigwedge_{i \in I} m_{i}(e)$ and $m_{i}^{\prime}(e)=\left(m_{i}(e)\right)^{\prime}$. In particular the top and the bottom elements in the lattice $\mathcal{M}$ are given respectively by $\mathcal{M}_{\top}(e)=1_{X} \forall e \in E$ and $\mathcal{M}_{\perp}(e)=0_{X} \forall e \in E$. 
Definition 4.4 Let $\psi: E \rightarrow F$ and $\varphi: X \rightarrow Y$ be two functions. Then the pair $(\psi, \varphi):(m, E, X) \rightarrow$ $(n, F, Y)$ is called a $L$-fuzzy soft mapping from $(m, E, X)$ to $(n, F, Y)$ if $\varphi \rightarrow \circ m \succeq n \circ \psi$ :

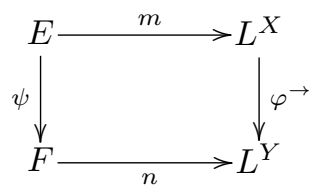

where $\varphi^{\rightarrow}: L^{X} \rightarrow L^{Y}$ is the forward $L$-powerset operator induced by the mapping $\varphi$ (see e.g. [18]), that is $\varphi^{\rightarrow}(A):=\varphi(A)$ for every $A \in L^{X}$.

The composition of two $L$-fuzzy soft mappings is defined component-wise, as in the case of soft mappings, ( we do not go into details here.) In the result we obtain the category of $L$-fuzzifying soft sets which will be denoted FSOFSET(2).

Let $(m, E, X)$ and $(n, F, Y)$ be two $L$-fuzzy soft sets and $(\psi, \varphi)$ be an $L$-fuzzy soft function from $(m, E, X)$ to $(n, F, Y)$.

(1) The image of $(m, E, X)$ under the $L$-fuzzy soft mapping $(\psi, \varphi)$ is the $L$-fuzzy soft set over $Y$ defined by $(\psi, \varphi)(m, E, X)=(\varphi(m), \psi(E), Y)$, where $\psi(E)$ is the image of $E$ in the category SET and

$$
\varphi^{\rightarrow}(m)_{k}(y)= \begin{cases}\bigvee_{\varphi(x)=y} \bigvee_{\psi(e)=k} m_{e}(x), & \text { if } \varphi(x)=y ; \\ 0, & \text { otherwise. }\end{cases}
$$

$\forall k \in \psi(E), \forall y \in Y$.

(2) The pre-image of $(n, F, Y)$ under the fuzzy soft function $(\psi, \varphi)$ is the $L$-fuzzy soft set over $X$ defined by $(\psi, \varphi)^{-1}(n, F, Y)=\left(\varphi^{-1}(n), \psi^{-1}(F), X\right)$, where $\psi^{-1}(F)$ is the pre-image of $F$ in the category of sets and

$$
\varphi^{-1}(n)_{f}(x)=n_{\psi(f)}(\varphi(x)), \forall f \in \psi^{-1}(F), \forall x \in X,
$$

( for the case $L=I$ see [2])

\section{2. $L$-fuzzifying soft neighborhood sets}

Further, in this section, we take a set $X$ in the role of the set of parameters and its powerset $2^{X}$ in the role of the set over which the $L$-fuzzy soft structure is constructed.

Definition 4.5 Let $\left(\mathcal{U}, X, 2^{X}\right)$ be an $L$-fuzzy soft set, that is $\mathcal{U}: X \rightarrow L^{2^{X}}$ is an $L$-fuzzy soft structure on the exponent $2^{X}$. We call $\mathcal{U}: X \rightarrow L^{2^{X}}$ an L-fuzzifying soft neighborhood structure and the corresponding triple $\left(\mathcal{U}, X, 2^{X}\right)$ an $L$-fuzzifying soft neighborhood set if for every $x \in X$ the family of functions $\mathfrak{U}=\left\{\mathcal{U}(x)=\mathcal{U}_{x}: 2^{X} \rightarrow L\right\}$ satisfies the following conditions:

(N1) $\mathcal{U}_{x}(A)>0$ implies $x \in A$;

(N2) $A \subseteq B \Longrightarrow \mathcal{U}_{x}(A) \leq \mathcal{U}_{x}(B)$;

(N3) $\mathcal{U}_{x}(A \cap B)=\mathfrak{U}_{x}(A) \wedge \mathcal{U}_{x}(B)$;

(N4) $\mathcal{U}_{x}(A)=\bigvee_{x \in A \subseteq B} \bigwedge_{y \in B} \mathcal{U}_{y}(B)$.

for all $x \in X, A, B \in 2^{X}$.
By NFSOFSET(2) we denote the full subcategory of the category FSOFTSET(2) whose objects are $L$-fuzzifying soft neighborhood sets.

In the next theorem we characterize $L$-fuzzifying topological spaces ([7], [21]) by means of $L$ fuzzifying soft neighborhood sets.

Theorem 4.6 Let $(X, \mathcal{T})$ be an L-fuzzifying topological space. We define a mapping $\mathcal{U}_{\mathcal{T}}: X \rightarrow L^{2^{X}}$ by setting $\mathcal{U}_{\mathcal{T}}(x)(A)=\bigvee_{x \in B \subset A} \mathcal{T}(B)$ for all $x \in$ $X, A \subseteq X$. Then $\left(\mathcal{U}_{\mathcal{T}}, X, 2^{\bar{X}}\right)$ is an L-fuzzifying soft neighborhood set. We call the L-fuzzifying soft neighborhood structure $\mathcal{U}_{\mathcal{T}}$ generated by the $L$ fuzzifying topology $\mathcal{T}$, and the triple $\left(\mathcal{U}_{\mathcal{T}}, X, 2^{X}\right)$ an $L$-fuzzifying soft set generated by the $L$-fuzzifying topological space $(X, \mathcal{T})$.

Conversely, given an L-fuzzifying soft neighborhood set $\left(\mathcal{U}, X, 2^{X}\right)$ we define a mapping $\mathcal{T}_{\mathcal{U}}: 2^{X} \rightarrow$ $L$ by setting $\mathcal{T}_{\mathcal{U}}(A)=\bigwedge_{x \in A} \mathcal{U}_{x}(A)$ for every $A \subseteq$ $X$. Then $\mathcal{T}_{\mathcal{U}}$ is an L-fuzzifying topology on $X$ called generated by the L-fuzzifying soft structure $\mathcal{U}: X \rightarrow L^{2^{X}}$.

Besides $\mathcal{T}_{\mathcal{U}_{\mathcal{T}}}=\mathcal{T}$ and $\mathcal{U}_{\mathcal{T}_{\mathcal{U}}}=\mathcal{U}$.

Proposition 4.7 Let $\left(\mathcal{U}, X, 2^{X}\right)$ and $\left(\mathcal{V}, Y, 2^{Y}\right)$ be two L-fuzzifying soft neighborhood sets. Then $(\psi,(\psi \rightarrow) \rightarrow):\left(\mathcal{U}, X, 2^{X}\right) \rightarrow\left(\mathcal{V}, Y, 2^{Y}\right)$ is an Lfuzzy soft mapping from $\left(\mathcal{U}, X, 2^{X}\right)$ to $\left(\mathcal{V}, X, 2^{X}\right)$ if and only if for all $x \in X$ and all $V \subset 2^{Y}$, $\mathcal{U}_{x}\left(\psi^{\rightarrow}\right)^{\leftarrow}(V) \geqslant \mathcal{V}_{\psi(x)}(V)$.

From Theorem 4.6 and Proposition 4.7 we get

Theorem 4.8 Functor

$$
\Phi: \operatorname{FTOP}(2) \rightarrow \operatorname{NFSOFSET}(2)
$$

which assigns to an L-fuzzifying topological space $(X, \mathcal{T})$ the L-fuzzifying soft neighborhood set $\left(\mathcal{U}_{\mathcal{T}}, X, 2^{X}\right)$ and assigns to a continuous mapping $\psi:\left(X, \mathcal{T}_{X}\right) \rightarrow\left(Y, \mathcal{T}_{Y}\right)$ the L-fuzzy soft mapping $(\psi,(\psi \rightarrow) \rightarrow):\left(\mathcal{U}_{\mathcal{T}_{X}}, X, 2^{X}\right) \rightarrow\left(\mathcal{U}_{\mathcal{T}_{Y}}, Y, 2^{Y}\right)$ is an isomorphism.

Let $\Psi: \operatorname{NFSOFSET}(2) \rightarrow \operatorname{FTOP}(2)$ denote the functor which is the inverse of $\Phi$.

\section{L-soft neigborhood set}

Recall that an $L$-fuzzy point with support $x \in X$ and value $\lambda \in L$ in a set $X$ is a mapping $x_{\lambda}: X \rightarrow L$ such that

$$
x_{\lambda}(y)= \begin{cases}\lambda, & \text { if } x=y \\ 0, & \text { otherwise }\end{cases}
$$

The set of all fuzzy points is denoted by $\operatorname{Pt}\left(L^{X}\right)$. Obviously $\operatorname{Pt}\left(L^{X}\right)=X \times M(L)$.

Definition 5.1 [10] Let $x_{\lambda} \in P t\left(L^{X}\right)$ and $u \in L^{X}$. If $\lambda \nless u^{\prime}(x)$, where $u^{\prime}(x)=(u(x))^{\prime}$, we say that $x_{\lambda}$ is quasi-coincident with $u$ and denote by $x_{\lambda} \widehat{q} u$. 
For every $x_{\lambda} \in \operatorname{Pt}\left(L^{X}\right)$ let $\overrightarrow{x_{\lambda}}$ denote the prefilter $\left\{v \in L^{X} \mid x_{\lambda} \widehat{q} v\right\}$. If $u \in L^{X}$ is such that $x_{\lambda} \widehat{q} u$ then by $\overrightarrow{x_{\lambda}} \mid u$ we denote the collection $\left\{v \in L^{X} \mid v \leqslant\right.$ $u$ and $x_{\lambda} \widehat{q} v$.

In this section, we take $X \times M(L)$ in the role of the set of parameters and the $L$-powerset $L^{X}$ as the set over which the soft structure is constructed.

Definition 5.2 Let $\left(\mathcal{U}, X \times M(L), L^{X}\right)$ be a soft set, i.e. $\mathcal{U}: X \times M(L) \rightarrow 2^{L^{X}}$. We call $\mathcal{U}: X \times$ $M(L) \rightarrow 2^{L^{X}}$ an L-soft neighborhood structure on the set $X$ and the triple $\left(\mathcal{U}, X \times M(L), L^{X}\right)$ an $L$ soft neighborhood set, if the family $\left\{\mathcal{U}\left(x_{\lambda}\right) \mid x_{\lambda} \in\right.$ $\left.\operatorname{Pt}\left(L^{X}\right)\right\} \subseteq 2^{L^{X}}$ satisfies the following conditions:

(N1) If $u \in \mathcal{U}_{x_{\lambda}}$, then $x_{\lambda} \widehat{q} u$;

(N2) If $u \in \mathcal{U}_{x_{\lambda}}$ and $u \leqslant v$, then $v \in \mathcal{U}_{x_{\lambda}}$;

(N3) If $u, v \in \mathcal{U}_{x_{\lambda}}$, then $u \wedge v \in \mathcal{U}_{x_{\lambda}}$;

(N4) For every $u \in \mathcal{U}_{x_{\lambda}}$, there exist $v \in \mathcal{U}_{x_{\lambda}}$ such that $v \leqslant u$ and $v \in \mathcal{U}_{y_{\alpha}}$ for all $y_{\alpha} \widehat{q} v$.

Let $\operatorname{NSOFSET}(L)$ denote the (full) subcategory of the category SOFSET whose objects are $L$-soft neighborhood sets.

In the next theorem we characterize $L$-topological spaces in the sense of Chang-Goguen ([4], [6]) by means of $L$-soft neighborhood sets.

Given an $L$-topological space $(X, T)$ and an $L$ fuzzy point $x_{\lambda}$ in it, an $L$-set $u \in L^{X}$ is called a quasi-co-neighborhood of $x_{\lambda}$ if there exists $v \leq u$ such that $v \in T$ and $x_{\lambda} \widehat{q} v$ [17], [10]. Let $\mathcal{Q}_{x_{\lambda}}$ stand for the family of all quasi-co-neighborhoods of an $L$-fuzzy point $x_{\lambda}$.

Theorem 5.3 Given an L-topological space $(X, \mathcal{T})$ and $x_{\lambda} \in \operatorname{Pt}\left(L^{X}\right)$, let $\mathcal{Q}_{x_{\lambda}}$ be the family of all quasico-neighborhoods of the fuzzy point $x_{\lambda}$ in this space. Further, define the mapping $\mathfrak{U}_{\mathcal{T}}: X \times M(L) \rightarrow 2^{L^{X}}$ by $\mathcal{U}_{\mathcal{T}}\left(x_{\lambda}\right)=\mathcal{Q}_{x_{\lambda}}$. Then $\left(\mathcal{U}_{\mathcal{T}}, X \times M(L), L^{X}\right)$ is an L-soft neighborhood set. We call it generated by the $L$-topology $\mathcal{T}$. Conversely, given an $L$-soft neighborhood set $\left(\mathcal{U}, X \times M(L), L^{X}\right)$ we define the family $\mathcal{T}_{\mathcal{u}}$ on $X$ by taking all $u \in L^{X}$ such that $u \in$ $\mathcal{U}\left(x_{\lambda}\right)$ whenever $x_{\lambda} \widehat{q} u$. Then $\mathcal{T}_{\mathcal{u}}$ is an L-topology on $X$ and $\left\{\mathcal{U}\left(x_{\lambda}\right): x_{\lambda} \in \operatorname{Pt}\left(L^{X}\right)\right\}$ is the quasi-coneighborhood system for the L-topology $\mathcal{T}_{\mathfrak{U}}$. We call the $L$-topological space $\left(X, \mathcal{T}_{\mathcal{U}}\right)$ generated by the $L$ soft neighborhood set $\left(\mathcal{U}, X \times M(L), L^{X}\right)$.

Besides $\mathcal{U}_{\mathcal{T}_{\mathcal{U}}}=\mathcal{U}$ and $\mathcal{T}_{\mathcal{U}_{\mathcal{T}}}=\mathcal{T}$.

Proposition 5.4 Let $\left(\mathcal{U}, X \times M(L), L^{X}\right)$ and $\left(\mathcal{V}, Y \times M(L), L^{Y}\right)$ be two L-soft neighborhood sets. The pair $(\psi,(\psi \rightarrow) \rightarrow):\left(\mathcal{U}, X \times M(L), L^{X}\right) \rightarrow$ $\left(\mathcal{V}, Y \times M(L), L^{Y}\right)$ is an L-soft mapping if and only if for all $x_{\lambda} \in \operatorname{Pt}\left(L^{X}\right)$ and all $u \in \mathcal{V}\left(\psi^{\rightarrow}\left(x_{\lambda}\right)\right)$, it holds $\left(\psi^{\rightarrow}\right)^{\leftarrow}(u) \in \mathcal{U}\left(x_{\lambda}\right)$ where $\psi^{\rightarrow}\left(x_{\lambda}\right)=\psi^{\rightarrow}(x)_{\lambda}$.

From the above proposition we get

Theorem 5.5 A pair

$\left(\psi,\left(\psi^{\rightarrow}\right) \rightarrow\right):\left(\mathcal{U}, X \times M(L), L^{X}\right) \rightarrow\left(\mathcal{V}, Y \times M(L), L^{Y}\right)$ is an L-soft map if and only if the mapping of the corresponding L-topological spaces

$$
\psi:\left(X, T_{U}\right) \rightarrow\left(Y, T_{\mathcal{V}}\right)
$$

is continuous.

We summarize the obtained results in the following

Theorem 5.6 Let a lattice $L$ be fixed. By assigning to an L-topological space $(X, \mathcal{T})$ an L-soft neighborhood set $\left(\mathcal{U}_{\mathcal{T}}, X \times M(L), L^{X}\right)$ and assigning to a continuous mapping $\varphi:\left(X, T_{U}\right) \rightarrow\left(Y, T_{V}\right)$ the soft mapping

$\left(\psi,\left(\psi^{\rightarrow}\right) \rightarrow\right):\left(\mathcal{U}, X \times M(L), L^{X}\right) \rightarrow\left(\mathcal{V}, Y \times M(L), L^{Y}\right)$

we get a functor $\Phi: \mathbf{T O P}(L) \rightarrow \operatorname{NSOFSET}(L)$ which establishes isomorphism between the category of $L$-topological spaces $\mathbf{T O P}(L)$ and the category of $L$-soft neighborhood spaces $\operatorname{NSOFSET}(L)$.

By $\Psi: \operatorname{NSOFSET}(L) \rightarrow \operatorname{TOP}(L)$ we denote the functor which is the inverse to the functor $\Phi$

\section{L-Fuzzy soft neigborhood set}

In this section, again we take $X \times M(L)$ in the role of the set of parameters and the $L$-powerset $L^{X}$ in the role of the set over which the $L$-fuzzy soft structure is constructed.

Definition 6.1 Let $\left(\mathcal{U}, X \times M(L), L^{X}\right)$ be an $L$ fuzzy soft set on $L^{X}$. We call $\mathcal{U}: X \times M(L) \rightarrow L^{L^{X}}$ an L-fuzzy soft neighborhood structure and $(\mathcal{U}, X \times$ $\left.M(L), L^{X}\right)$ an $L$-fuzzy soft neighborhood set if for every $x_{\lambda} \in \operatorname{Pt}\left(L^{X}\right)$ the family $\mathcal{U}\left(x_{\lambda}\right)=\mathcal{U}_{x_{\lambda}} \in L^{L^{X}}$ satisfies the following conditions:

(N1) $\mathcal{U}_{x_{\lambda}}(u) \neq 0_{X}$ implies $x_{\lambda} \widehat{q} u$;

(N2) $u \leq v \Longrightarrow \mathcal{U}_{x}(u) \leq \mathcal{U}_{x}(v)$;

(N3) $\mathcal{U}_{x_{\lambda}}(u \wedge v)=\mathcal{U}_{x_{\lambda}}(u) \wedge \mathcal{U}_{x_{\lambda}}(v)$;

(N4) $\mathcal{U}_{x_{\lambda}}(u)=\bigvee_{v \in \overrightarrow{x_{\lambda}} \mid u} \bigwedge_{y_{\alpha} \widehat{q v}} \mathcal{U}_{y_{\alpha}}(v)$.

for all $x \in X, u, v \in L^{X}$.

By NFSOFSET(L) we denote the category of $L$-fuzzy soft neighborhood sets and in a natural way defined $L$-fuzzy soft mappings between them. (The size limitations does not allow us to go into details here).

In the next theorem we characterize $L$-fuzzy topological spaces ([9], [19], [20]) by means of $L$-fuzzy soft neighborhood sets.

Theorem 6.2 Given an L-fuzzy topological space $(X, \mathcal{T})$ we define the mapping $\mathcal{U}_{\mathcal{T}}: X \times M(L) \rightarrow$ $L^{L^{X}}$ by

$$
\mathcal{U}_{\mathcal{T}}\left(x_{\lambda}\right)(u)=\left\{\begin{array}{cc}
\bigvee_{v \in \overrightarrow{x_{\lambda}} \mid u} \mathcal{T}(v), & \text { if } x_{\lambda} \widehat{q u} u \\
0, & \text { if otherwise }
\end{array}\right.
$$


for every $x_{\lambda} \in \operatorname{Pt}\left(L^{X}\right), u \in L^{X}$. Then $\left(\mathcal{U}_{\mathcal{T}}, X \times\right.$ $\left.M(L), L^{X}\right)$ is an $L$-fuzzy soft neighborhood set. We call it generated by the $L$-fuzzy topology $\mathcal{T}$.

Conversely, given an L-fuzzy soft neighborhood set $\left(\mathcal{U}, X \times M(L), L^{X}\right)$ we define the mapping $\mathcal{T}_{\mathcal{U}}$ : $L^{X} \rightarrow L$ by the equality $\mathcal{T}(u)=\bigwedge_{x_{\lambda} \widehat{q} u} \mathcal{U}_{x_{\lambda}}(u)$. Then $\mathcal{T}_{\mathcal{u}}$ is an L-fuzzy topology on $X$. We call the $L$-fuzzy topological space $\left(X, \mathcal{T}_{\mathcal{u}}\right)$ induced by the $L$ fuzzy soft neighborhood set $\left(\mathcal{U}, X \times M(L), L^{X}\right)$.

Besides $\mathcal{U}_{\mathcal{T}_{\mathcal{u}}}=\mathcal{U}$ and $\mathcal{T}_{\mathcal{U}_{\mathcal{T}}}$.

Proposition 6.3 Let $\left(U, X \times M(L), L^{X}\right)$ and $\left(\mathcal{V}, Y \times M(L), L^{Y}\right)$ be two L-fuzzy soft neighborhood sets. Then

$\left(\psi,\left(\psi^{\rightarrow}\right) \rightarrow\right):\left(\mathcal{U}, X \times M(L), L^{X}\right) \rightarrow\left(\mathcal{V}, Y \times M(L), L^{Y}\right)$

is an L-fuzzy soft mapping if and only if for all $x_{\lambda} \in$ $P t\left(L^{X}\right)$ and all $u \in L^{Y}$, it holds

$$
\mathcal{U}_{x_{\lambda}}\left(\psi^{\leftarrow}(u)\right) \geqslant \mathcal{V}_{\psi(x)_{\lambda}}(u)
$$

From this proposition we easily obtain the next

Theorem 6.4 Let $\psi: X \rightarrow Y$ be a mapping. Then

$\left(\psi,\left(\psi^{\rightarrow}\right) \rightarrow\right):\left(\mathcal{U}, X \times M(L), L^{X}\right) \rightarrow\left(\mathcal{V}, Y \times M(L), L^{Y}\right)$

is a soft mapping if and only if the mapping of the corresponding L-fuzzy topological spaces $\psi$ : $\left(X, \mathcal{T}_{\mathcal{U}}\right) \rightarrow\left(Y, \mathcal{T}_{\mathcal{V}}\right)$ is continuous.

From the above results we have the following.

Theorem 6.5 Let a lattice $L$ be fixed. By assigning to an L-fuzzy soft neighborhood set $(\mathcal{U}, X \times$ $\left.M(L), L^{X}\right)$ the L-fuzzy topological space $\left(X, \mathcal{T}_{\mathcal{U}}\right)$ and assigning to a soft mapping $\left(\psi,\left(\psi^{\prime}\right) \rightarrow\right)$ : $\left(\mathcal{U}, X \times M(L), L^{X}\right) \rightarrow\left(\mathcal{V}, Y \times M(L), L^{Y}\right)$ of $L$ fuzzy soft neighborhood sets the (continuous) mapping $\psi:\left(X, \mathcal{T}_{\mathcal{U}}\right) \rightarrow\left(Y, \mathcal{T}_{\mathcal{V}}\right)$ of the corresponding L-fuzzy topological spaces we get a functor $\Psi$ : $\operatorname{NFSOFSET}(L) \rightarrow \operatorname{FTOP}(L)$ which establishes isomorphism between the category of L-fuzzy topological spaces $\mathbf{F T O P}(L)$ and the category of $L$-fuzzy soft neighborhood sets $\operatorname{NFSOFSET}(L)$.

Acknowledgements This research was done during the stay of the first named author at the University of Latvia from September 2010 till January 2011. This stay was organized in the frames of the Erasmus exchange programme between the University of Latvia and Kocaeli University. Banu Pazar Varol gratefully acknowledges financial support received from the Erasmus programme and from the Kocaeli University.

\section{References}

[1] U. Acar, F. Koyuncu and B. Tanay, Soft sets and soft rings, Computers and Mathematics with Applications, 59:3458-3463, 2010.
[2] A. Aygünoglu and H. Aygün, Introduction to fuzzy soft groups, Computers and Mathematics with Applications, 58:1279-1286, 2009.

[3] A. Aygünoglu and H. Aygün, Soft topological spaces, Submitted.

[4] C.L. Chang, Fuzyy topological spaces, J. Math. Anal. Appl., 24:182-190, 1968.

[5] D. Dubois, W. Ostasiewicz and H. Prade, Fuzzy sets: History and basic notions. In: D. Dubois and H. Prade (eds.), Fundamentals of Fuzzy Sets, pp. 947-1010. Kluwer Acad. Publ., Boston, Dodrecht, 1999.

[6] J.A. Goguen, L-Fuzzy sets, J. Math. Anal. Appl., 18:145-174, 1967.

[7] U. Höhle, Upper-semicontinuous fuzzy sets and applications, J. Math. Anal. Appl., 78:659-673, 1980.

[8] J.L. Kelly, General Topology, New York 1955.

[9] T. Kubiak, On fuzzy topologies, PhD Thesis, Adam Mickiewicz University, Poznan (Poland), 1985.

[10] Y.M. Liu and M.K. Luo, Fuzzy topology, World Scientific Publication, Singapore, 1998.

[11] P.K. Maji, R. Biswas and A.R. Roy, Fuzzy soft set, J. Fuzzy Math., 9(3):589-602, 2001.

[12] D. Molodtsov, Soft set theory - First results, Computers Math. Applic., 37(4/5):19-31, 1999.

[13] D. Molodtsov Theory of Fuzzy Sets Editorial URSS , Moscow 2004. In Russian

[14] B. Pazar Varol and H. Aygün, Fuzzy soft topological spaces and subspaces, Submitted.

[15] B. Pazar Varol, A. Aygünoglu and H. Aygün, On fuzzy soft rings, Submitted.

[16] D. Pei and D. Miao, From soft sets to information systems, Granular Computing, 2005 IEEE International Conference on,(2):617-621, 2005.

[17] P.M. Pu and Y.M. Liu, Fuzzy topology (I), Neighborhood structure of a fuzzy point and Moore-Smith converges, J. Math. Anal. Appl., 76:571-599, 1980.

[18] S.E. Rodabaugh, Powerset Operator Foundations For Poslat Fuzzy Set Theories and Topologies, Chapter 2 in Mathematics of Fuzzy Sets: Logic, Topology adn Measure Theory, U. Höhle and S.E. Rodabaugh eds., Kluwer Academic Publishers, 91-116, 1999.

[19] A.P. Shostak, On a fuzzy topological structure, Suppl. Rend. Circ. Matem. Palermo 2 Ser II, 11:89-103, 1985.

[20] A.P. Shostak, Two decades of fuzzy topology: Basic ideas, notions and results, Russian Math. Surveys, 44:125-186, 1989.

[21] Mingsheng Ying, A new approach to fuzzy topology (I), Fuzzy Sets and Systems, 39:303321, 1991. 